\title{
Robotic cans surface inspection system based on shape features
}

\begin{abstract}
Computer vision systems are one of the most widely used techniques in Automation and have been extensively used for industry automation. Industrial automation deals mainly with the automation of production, quality control and materials management processes. One trend is the increasing use of Machine vision to offer automatic inspection and robot guidance functions, while the other is a continued increase in the use of robots. The aim of this paper is to provide a robotic cans surface inspection system based on the shape. The proposed system is simple and user friendly yet accurate, uses Hu moment as a feature of detected shape in the image and compared to the range of acceptable $\mathrm{Hu}$ moment gained from training. It is composed of a camera attached to a PC with TCP/IP, image acquisition, analysis, and inspection implemented by Open CV Library for image processing. The method described in this paper checks on the statistical-based approaches for feature extraction such as moment feature as part of the final inspection system. Robotic arm is programed as a client server method to receive action and position from the $\mathrm{PC}$, which carries out the image processing as well.
\end{abstract}

Keyword: Robotics; Computer vision; Automation; Machine vision; OpenCV 